\title{
Optimal Brokerage Commissions for Fair Insurance: A First Order Approach
}

\author{
Arthur Hau \\ Lingnan University, Tuen Mun, New Territories, Hong Kong.
}

This paper studies a principal-agent insurance brokerage problem with a riskaverse principal (an insured) and a risk-neutral agent (a broker). The concept of "mean-preserving, spread-reducing" (MPSR) effort is introduced to model the broker's activities. Using the first-order approach, it is shown that under some common conditions, the insured may "concavify" the reward function to induce the risk-neutral agent to exert MPSR brokering effort. These conditions, together with an additional condition, guarantee the validity of the first-order approach even when the monotone likelihood ratio condition (used exclusively to justify the firstorder approach) is violated.

The Geneva Risk and Insurance Review (2011) 36, 189-201. doi:10.1057/grir.2010.11; published online 1 February 2011

Keywords: insurance brokerage commissions; principal-agent problem; maximum likelihood ratio condition; first-order approach; mean-preserving spread-reducing effort

\section{Introduction}

In their seminal paper, Spence and Zeckhauser (1971) analyze a principal-agent model in which an insurance company (the principal) alleviates the moral hazard problem of an insured (the agent), who has no incentive to exert self-protection effort. It is tempting to apply the results derived from the traditional principalagent problem (e.g., Spence and Zeckhauser, 1971; Campbell and Kracaw, 1987; Oyer, 2000) to the insurance brokerage problem. In fact, Cummins and Doherty (2006) conjectures that "principal-agent theory" may be used to analyze the inefficiency of fee or commission compensation schemes that currently prevail in insurance brokerage. In insurance broking, an insured (the principal) hires a broker (the agent) to arrange for insurance coverage for a certain potential loss.

Unfortunately, there is a major feature in insurance broking that distinguishes it from the principal-agent problem analyzed in the literature. Particularly, the principal is risk-neutral and the agent is risk-averse in the traditional principalagent problem, whereas the opposite is often true in insurance brokerage. According to Tommy (2000), the insurance brokerage market is dominated by several large brokers. Almost 60 percent of global insurance has been brokered by 
the largest two insurance brokers, namely, Marsh Inc. and Aon Corp. A large insurance broker can diversify in the financial market or has a sufficiently large portfolio of (independent) clients. Therefore, a large insurance broker may accept an insurance broking contract based on the expected value of the contract, acting as if it is risk-neutral. Another reason for an insurance broking company to be risk-neutral is that its shareholders can diversify by investing in broking companies and non-broking companies. On the other hand, the principal that purchases insurance may be a small enterprise or a company facing bankruptcy cost and can be considered as risk-averse.

This paper attempts to study the principal-agent problem with a risk-averse principal and a risk-neutral agent in which the agent's effort exhibits the "mean-preserving, spread reducing" (MPSR) property in the RothschildStiglitz sense. An immediate application of this general model is the case of an insurance broker helping an insured arrange for appropriate insurance policies or bargaining with insurance companies to tailor-make policies to obtain optimal actuarially fair coverage. Under the general model, the agent's activities reduce the spread of the potential loss of the principal keeping its mean unchanged. However, the agent has no incentive to take account of the principal's risk attitude. Therefore, the principal needs to design a reward (commission) profile to provide an appropriate incentive to the agent. The solution of the problem serves as a benchmark for checking whether existing broking fees and commission schedules are or are not optimal to the insured.

Finally, Mirrlees (1999/1975) points out that the first-order approach used by Spence and Zeckhauser (1971) for solving the traditional principal-agent problems is often invalid. Grossman and Hart (1983) and Rogerson (1985) show that if the principal's random outcome satisfies both the monotone likelihood ratio condition (MLRC) and the convexity of distribution function condition (CDFC), the first-order approach is valid. ${ }^{1}$ Jewitt (1988) replaces the CDFC by less restrictive conditions on the distribution function and imposes an additional restriction on the agent's utility function. The use of the firstorder approach for solving the insurance broking problem also needs to be justified.

Two questions will be answered in this paper: (i) What is the general shape of the optimal reward function under the insurance brokerage problem with a risk-averse principal, a risk-neutral agent, and MPSR agent's effort? (ii) Under what conditions is the first-order approach justified under the insurance brokerage problem? The paper is organized as follows. The next section states

\footnotetext{
${ }^{1}$ Whitt (1980) and Rogerson (1985) suggest that the MLRC implies that a rise in the agent's effort results in a first-order stochastic dominating change in the principal's outcome and hence a rise in the principal's average outcome.
} 
and solves the principal-agent insurance brokerage problem using the firstorder approach. The subsequent section derives the shape of the optimal reward function. The penultimate section derives the conditions that guarantee the validity of the first-order approach. The final section concludes.

\section{A principal-agent insurance brokerage model}

A risk-averse principal (an insured) faces an insurable risk, $\tilde{x}$. The principal hires a risk-neutral agent (an insurance broker) to search for appropriate insurance coverage. For convenience, this class of risk management problems will be called "insurance brokerage problems" from now on. Let $a \geqslant 0$ be the agent's effort with cost function $c$ satisfying $c(a)>0, c^{\prime}(a)>0, c^{\prime \prime}(a)>0, \forall a>0$ and $\lim _{a \rightarrow 0} c^{\prime}(a)=0 .{ }^{2}$ Assume $\tilde{x}$ has realizations $x$, support $[\underline{x}, \bar{x}]$, distribution function $F$, and density function $f:[x, \bar{x}] \times \mathfrak{R}_{+} \rightarrow[0,1]$ with $f(x, \bar{a})>0, \forall x \in[x, \bar{x}]$ and $\forall a$. Assume $f$ is twice continuously differentiable in $a$ and that both $f_{a}$ and $f$ are twice continuously differentiable in $x$. Let $s:[\underline{x}, \bar{x}] \rightarrow \mathfrak{R}_{+}$be the reward function.

Assume that the principal is incapable of observing $a$ (see, e.g., Holmstrom, 1979). This assumption may or may not hold in general. However, in property and liability insurance, the ultimate coverage received is often very complicated depending on a lot of factors, such as coinsurance rate, deductibles or excesses on different exposures, policy limits (e.g., per claim limits and aggregate limits), exclusions, and endorsements. It can be too difficult and costly for the insured to observe $a$. Therefore, the unobservability assumption seems reasonable. Alternatively, one may assume partial observability.

The principal is risk-averse with thrice differentiable utility function $v$ satisfying $v^{\prime}>0$ and $v^{\prime \prime}<0$. The principal's maximization problem is

$$
\max _{\{s(\cdot), a\}} \int_{\underline{x}}^{\bar{x}} v[x-s(x)] f(x, a) \mathrm{d} x
$$

subject to the risk-neutral agent's incentive compatibility (IC) constraint given by

$$
a \in \arg \max \int_{\underline{x}}^{\bar{x}} s(x) f(x, a) \mathrm{d} x-c(a) .
$$

${ }^{2}$ In insurance brokerage, to increase coverage, an insurance broker has to dedicate more resources and effort to search among different insurance policies offered in the market and to bargain with insurers to raise indemnity rates, to lower deductibles, to delete exclusions, or to add endorsements to broaden the coverage under a standard policy. An alternative interpretation is that the insured has many small independent insurable risk units such that the agent's cost rises as a higher percentage of these risk units receives coverage. 
The principal, therefore, chooses reward function $s$ that is used to "control" the agent's choice of $a$ via the agent's IC constraint. Here, the IC constraint simply says that given reward function $s$, the agent chooses $a$ optimally to maximize its net expected reward. In addition to the IC constraint, the principal is also subject to the agent's individual rationality constraint,

$$
\int_{\underline{x}}^{\bar{x}} s(x) f(x, a) \mathrm{d} x-c(a) \geqslant R,
$$

where $R$ is the agent's reservation utility.

According to the first-order approach, the IC constraint may be replaced by the first-order condition of the agent's maximization problem (2), namely, ${ }^{3}$

$$
\int_{\underline{x}}^{\bar{x}} s(x) f_{a}(x, a) \mathrm{d} x-c^{\prime}(a)=0 .
$$

Using the optimal control technique, the first-order conditions are given by:

$$
\begin{gathered}
v^{\prime}[x-s(x)]=\lambda+\mu \frac{f_{a}(x, a)}{f(x, a)} ; \\
\int_{\underline{x}}^{\bar{x}} v[x-s(x)] f_{a}(x, a) \mathrm{d} x+\mu\left[\int_{\underline{x}}^{\bar{x}} s(x) f_{a a}(x, a) \mathrm{d} x-c^{\prime \prime}(a)\right]=0 .
\end{gathered}
$$

To further investigate the optimal solution of the insurance brokerage problem, some assumptions should be made explicit. In particular, it is assumed that a unique interior solution of $a$ exists for the principal's problem. ${ }^{4}$ Moreover, it is necessary to specify the nature of the agent's effort. Let us focus on an actuarially fair or unbiased risk transfer under which a rise in coverage

${ }^{3}$ Araujo and Moreira (2001) show how the traditional principal-agent problem can be solved without using the first-order approach.

${ }^{4}$ Notice that even if it is assumed that $\lim _{a \rightarrow 0} c^{\prime}(a)=0$, the risk-neutral agent may not exert $a>0$ when the agent's effort is MPSR, as will be defined below, depending on the reward function. Particularly, the readers can check that when $\mu=0$ and $s(x)=x-k$, where $k$ is a constant, (4) $-(6)$ are satisfied at $a=0$. By integration by parts, with an MPSR effort, (4) holds even when $a=0$. 
reduces the spread of the random outcome without changing its mean in the Rothschild-Stiglitz sense.

Definition An agent's effort is said to be MPSR if $\int_{\underline{x}}^{x} F_{a}(\theta, a) \mathrm{d} \theta<0, \forall a$ and $\forall x \in(\underline{x}, \bar{x})$ and $\int_{\underline{x}}^{\bar{x}} F_{a}(\theta, a) \mathrm{d} \theta=0, \forall a .^{5}$

Pick any $\Delta a>0$. For all $x \in(\underline{x}, \bar{x})$, integrating $\int_{\underline{x}}^{x} F_{a}(\theta, a) \mathrm{d} \theta<0$ from $a$ to $a+\Delta a$ gives $\int_{\underline{x}}^{x}[F(\theta, a)-F(\theta, a+\bar{\Delta} a)] \mathrm{d} x>0$. Similarly, $\int_{\underline{x}}^{\bar{x}} F_{a}(\theta, a) \mathrm{d} \theta=0$ implies $\int_{\underline{x}}^{\bar{x}}[F(\theta, a)-F(\theta+\Delta a)] \mathrm{d} \theta=0$. Now, for any two distributions $G(\theta)$ and $H(\theta), G$ is a Rothschild-Stiglitz mean-preserving spread of $H$ if $\int_{\underline{x}}^{x}[H(\theta)-G(\theta)] \mathrm{d} \theta>0$, $\forall x \in[\underline{x}, \bar{x})$ and $\int_{\underline{x}}^{\bar{x}}[H(\theta)-G(\theta)] \mathrm{d} \theta=0$. Letting $G(\theta)=F(\theta, a+\Delta a)$ and $H(\theta)=F(\theta, a)$ certainly yields that any increase in $a$ satisfying the MPSR definition preserves the mean while reduces the spread of the random outcome in the RothschildStiglitz sense.

Clearly, if an insurance broker's effort is to raise the indemnity rate of a fair coinsurance-type insurance policy, it should satisfy the MPSR condition. This is also true for a rise in insurance deductible or policy limit and for a risk management department that hedges a higher percentage of the company's exchange rate or price risk by purchasing more commodity forwards or in the future. Let $w$ be the initial wealth of the principal and $\tilde{y}$ be an insurable risk (with density function $h$, distribution function $H$, and support $[\underline{y}, \bar{y}]$ ) faced by the principal. The insurance broker exerts effort to raise indemnity rate $a \in[0,1]$ of a fair coinsurance-type policy such that the principal's net worth equals

$$
w-\tilde{y}+a(\tilde{y}-E \tilde{y})-s(\tilde{x}),
$$

where $a E \tilde{y}$ is the fair premium. Let $\tilde{x}=w-\tilde{y}+a(\tilde{y}-E \tilde{y})$. The support of $\tilde{x},[\underline{x}, \bar{x}]$, now equals $[w-\bar{y}+a(\bar{y}-E \tilde{y}), w-\underline{y}+a(\underline{y}-E \tilde{y})]$. The principal's net payoff is exactly $\tilde{x}-s(\tilde{x})$.

To see that the distribution of $\tilde{x}$ satisfies the MPSR condition, notice that

$$
F(x, a)=\int_{\underline{x}}^{x} f(x, \theta) \mathrm{d} \theta=\int_{y}^{\bar{y}} h(\xi) \mathrm{d} \xi=\int_{\frac{w-x-a E \tilde{y}}{1-a}}^{\bar{y}} h(\xi) \mathrm{d} \xi
$$

${ }^{5}$ The MPSR concept can be specified as non-strict satisfying $\int_{\underline{x}}^{x} F_{a}(\theta, a) \mathrm{d} \theta \leqslant 0, \forall a$ and $\forall x \in(\underline{x}, \bar{x})$ with $\int_{x}^{x} F_{a}(\theta, a) \mathrm{d} \theta<0, \forall a$ for some subsets of $(\underline{x}, \bar{x})$ having positive measures, and $\int_{\underline{x}}^{\bar{x}} F_{a}(\theta, a) \mathrm{d} \theta=0$. 
such that when $a<1$,

$$
F_{a}(x, a)=-(1-a)^{-2}(w-x-E \tilde{y}) \cdot h\left(\frac{w-x-a E \tilde{y}}{1-a}\right) .
$$

Now, $F_{a}(\bar{x}, a)=0$. The right side of (7) changes from negative to positive (as $-(w-x-E \tilde{y})$ changes from negative to positive) when $x$ increases. This certainly implies that the MPSR condition is satisfied as $\int_{x}^{x} F_{a}(\theta, a) \mathrm{d} \theta<0$ for all $x \in(\underline{x}, \bar{x})$ because $\int_{\underline{x}}^{\bar{x}} F_{a}(x, a) \mathrm{d} \underline{x}=0$. The last equality is due to

$$
\int_{\underline{x}}^{\bar{x}} x f(x, a) \mathrm{d} x=E \tilde{x}=E[w-\tilde{y}+a(\tilde{y}-E \tilde{y})]=w-E \tilde{y}
$$

regardless of the value of $a$ such that

$$
\begin{aligned}
0= & \frac{\mathrm{d} E \tilde{x}}{\mathrm{~d} a}=\int_{\underline{x}}^{\bar{x}} x f_{a}(x, a) \mathrm{d} x=\bar{x} F_{a}(\bar{x}, a) \\
& -\int_{\underline{x}}^{\bar{x}} F_{a}(x, a) \mathrm{d} x=-\int_{\underline{x}}^{\bar{x}} F_{a}(x, a) \mathrm{d} x .
\end{aligned}
$$

\section{The shape of the optimal reward function}

Intuition suggests that the optimal reward function should create some spread in the agent's net reward, so that the agent will take the principal's risk attitude into account when exerting MPSR effort. Before investigating the shape of the optimal reward function, it is necessary to first check that multipliers $\lambda$ and $\mu$ are strictly positive.

Lemma Suppose the agent's effort is MPSR and $a$ has an interior solution. When the principal is risk-averse and the agent is risk-neutral, $\lambda>0$ and $\mu>0$.

Proof Interested readers can obtain the proof from the author on request. The first task is to compare the shape of the reward function of the insurance brokerage problem with that of the traditional principal-agent problem. It is well-known (see, e.g., Jewitt, 1988) that the reward function and the $f_{a} / f$ ratio 
move in the same direction as $x$ increases under the traditional problem. When $f_{a} / f$ is monotonically increasing satisfying the MLRC (to be defined formally in the next section), so is the reward function.

Denote the Arrow-Pratt coefficient of absolute risk aversion for utility function $v$ by $A_{v}=-v^{\prime \prime} / v^{\prime} . v$ is said to exhibit decreasing absolute risk aversion if $A_{v^{\prime}}<0$, non-increasing absolute risk aversion (NIARA) if $A_{v^{\prime}} \leqslant 0$, and constant absolute risk aversion (CARA) if $A_{v^{\prime}}=0$. It is generally believed that NIARA is consistent with many reasonable economic behaviours (see, e.g., Arrow, 1963; Mossin, 1968). The following theorem states the relation between the principal's net payoff (i.e., $x-s(x))$ and the $f_{a} / f$ ratio, and the shape of reward function $s$ :

Theorem 1 Suppose the principal is risk-averse and the agent is risk-neutral.

(a) $1-s^{\prime}(x)$ and $\left(f_{a} / f\right)_{x}$ have opposite signs.

(b) If $v$ exhibits NIARA and $\left(f_{a} / f\right)_{x x}<0, \forall x \in[\underline{x}, \bar{x}]$, then $s^{\prime \prime}(x)<0{ }^{6}$

Proof See Appendix.

Theorem 1(a) suggests that the principal's optimal net return, $x-s(x)$, and the $f_{a} / f$ ratio move in opposite directions as $x$ increases, whereas the agent's optimal reward $s(x)$ may rise or fall when $f_{a} / f$ rises. This result is obviously different from that of the traditional problem in which the risk-averse agent's optimal reward and the $f_{a} / f$ ratio move in the same direction.

Theorem 1(b) suggests that the reward function $s$ is concave in $x$ whenever $f_{a} / f$ is concave in $x$ and the principal's utility function exhibits NIARA. Theorem 1(b) is intuitive. To induce the risk-neutral insurance broker to exert costly effort to search for appropriate insurance coverage to reduce the risk, the insured should concavify the broker's payoff function so that the broker will act as if it is risk-averse. It will be apparent in the next section that the conditions stated in Theorem 1(b) are important for justifying the first-order approach for the insurance brokerage problem because $s^{\prime \prime}<0$ guarantees that the agent's expected payoff is concave in $a$.

\section{Justifying the first-order approach to the insurance brokerage problem}

As pointed out by Mirrlees (1975), the first-order approach to principal-agent problems may not be valid in general. Rogerson (1985) justifies the first-order

\footnotetext{
${ }^{6}$ The assumption of $\left(f_{a} / f\right)_{x x}<0, \forall x \in[\underline{x}, \bar{x}]$ can be replaced by $\left(f_{a} / f\right)_{x x} \leqslant 0$ with $\left(f_{a} / f\right)_{x x}=0$ iff $\left(f_{a} / f\right)_{x} \neq 0, \forall x \in[\underline{x}, \bar{x}]$.
} 
approach to the traditional principal-agent problems by using the following conditions:

Definition An agent's effort is said to satisfy the MLRC if $\left(f_{a} / f\right)_{x} \geqslant 0$, $\forall x \in[\underline{x}, \bar{x}]$ and $\left(f_{a} / f\right)_{x}>0$ for some subsets of $[\underline{x}, \bar{x}]$ with positive measures. ${ }^{7}$

Definition An agent's effort is said to satisfy the CDFC if $F_{a a}(x, a) \geqslant 0$ for all $a$ and all $x \in[\underline{x}, \bar{x}]$.

Jewitt $(1988$, p. 1177) gives a simple example to argue that "most of the distributions commonly occurring in statistics (and economics) do not have the CDF property". He maintains the MLRC, but relaxes the CDFC by imposing other restrictions. ${ }^{8}$ Later, LiCalzi and Spaeter (2003) show that some reasonable classes of distributions actually satisfy both the MLRC and the CDFC.

The following states that the MLRC contradicts with the MPSR condition so that Rogerson's (1985) and Jewitt's (1988) conditions cannot be used to justify the first-order approach under the insurance brokerage problem.

Claim The MPSR condition and the MLRC cannot hold simultaneously.

\section{Proof See Appendix.}

The pertinent question is whether the first-order approach can be justified under the insurance brokerage problem in which the principal is risk-averse, the agent is risk-neutral, and the agent's effort is MPSR. The following theorem provides an answer to the question:

Theorem $2^{9}$ Suppose the principal is risk-averse, the agent is risk-neutral, and the agent's effort is MPSR. The first-order approach is valid such that an

${ }^{7}$ The literature often defines the MLRC such that the distribution of the random outcome satisfies $\left(f_{a} / f\right)_{x} \geqslant 0, \forall x \in[x, \bar{x}]$ (see, e.g., Rogerson, 1985, p. 1361; Jewitt, 1988, condition 2.11). The readers can verify that in the special case with $\left(f_{a} / f\right)_{x}=0, \forall x \in[\underline{x}, \bar{x}], a>0$ cannot be optimal under the traditional principal-agent problem.

${ }^{8}$ Sinclair-Desgagné (1994) also justifies the first-order approach for the multi-signal principalagent problem by maintaining the MLRC. Brown et al. (1986) do not utilize the MLRC in part (i) of their theorem, whereas the other three parts of their theorem all assume that the MLRC holds. However, part (i) of Brown et al.'s (1986) theorem states that the first-order approach is valid when the risk-averse agent's utility is sufficiently concave without stating how concave the agent's utility function really needs to be.

${ }^{9}$ One can replace condition (a) by $\int_{\underline{x}}^{x} F_{a a}(\theta, a) \mathrm{d} \theta \geqslant 0, \forall a$ and $\forall x \in[\underline{x}, \bar{x}]$ with $\int_{x}^{x} F_{a a}(\theta, a) \mathrm{d} \theta>0$ for some subsets of $[x, \bar{x}]$ with positive measures. Moreover, one can replace condition (b) by $\left(f_{a} / f\right)_{x x} \leqslant 0$ with $\left(\bar{f}_{a} / f\right)_{x x}=0$ iff $\left(f_{a} / f\right)_{x} \neq 0, \forall a$ and $\forall x \in[\underline{x}, \bar{x}]$. 
interior solution for $a$ can be sustained by the agent's first-order condition and can be optimal to the agent's problem if

(a) $\int_{x}^{x} F_{a a}(\theta, a) d \theta>0, \forall a$ and $\forall x \in[\underline{x}, \bar{x}]$,

(b) $\left(\bar{f}_{a} / f\right)_{x x}<0, \forall a$ and $\forall x \in[x, \bar{x}]$, and

(c) $v$ exhibits NIARA.

Proof See Appendix.

Theorem 2 presents a new set of conditions that justifies the first-order approach without using either the MLRC or the CDFC. These conditions are simpler than those of Jewitt (1988). Two reasons for such simplification are (i) the replacement of risk-neutral principals/risk-averse agents by risk-averse principals/risk-neutral agents and (ii) the nature of MPSR activities giving rise to $F_{a}(\bar{x}, a)=0$. Notice that condition (a) of Theorem 2 is also used by Jewitt (1988, Theorem 2) to replace the CDFC. Condition (b) in its strict or non-strict form is satisfied by some commonly encountered distribution functions, such as normal, truncated normal, exponential, and some members of generalized gamma distributions, under the coinsurance-type insurance problem discussed in the section "A principal-agent insurance brokerage model". Condition (c) is similar to but less complicated than condition (2.12) of Jewitt's Theorem 2 (1988), namely, $\omega^{\prime \prime}(z) \leqslant 0, \forall z>0$, where $\omega(z)=u\left[u^{\prime-1}(1 / z)\right]$. According to Jewitt, his condition (2.12) essentially requires that the coefficient of absolute risk aversion of the agent's utility must not decrease too quickly.

The intuition of Theorem 2 becomes apparent when one observes from Theorem 1 that conditions (b) and (c) of Theorem 2 imply that reward function $s$ is concave. The concavity of $s$ in $x$ renders the agent's expected payoff concave in $a$ as shown in the proof of Theorem 2. With concave expected payoff, the risk-neutral agent is induced to exert optimal MPSR effort as if it is risk-averse (or the agent is simply forced to take account of the risk-averse attitude of the principal as a result of the concave reward function). Notice from the proof of Theorem 2 (particularly Eq. (15)) that a constant or a linear reward function cannot be optimal because $a>0$ cannot be sustained by the agent's first-order condition when $s^{\prime \prime}=0$.

Remark In general, an agent (e.g., a risk manager) may be risk-averse. Allowing for risk-averse agents makes the insurance brokerage problem more complicated. To yield results similar to those stated in Theorems 1 and 2 , one needs to impose restrictions (such as CARA) on the utility functions of both the principal and the agent. Moreover, one needs to explicitly assume $\mu>0$ as the inequality is no longer guaranteed. Interested readers can obtain additional results for the case with risk-averse agents from the author on request. 


\section{Conclusion}

This paper has analysed the principal-agent insurance brokerage problem by introducing the concept of "mean-preserving, spread-reducing" effort. It has been shown that when the principal's utility exhibits NIARA, the concavity of the $f_{a} / f$ ratio gives rise to a concave optimal reward function inducing the risk-neutral agent to exert MPSR effort. The same conditions together with an additional condition on the distribution function of the risk (similar but simpler than those imposed by Jewitt, 1988) guarantee the validity of the first-order approach, without using either the MLRC or the CDFC.

In reality, however, a reward function that is concave in the random outcome is seldom observed in insurance brokering. There are several possible explanations. First, the insurance brokering market may face market imperfection and market concentration (see, e.g., Tommy, 2000) so that brokerage commissions may be set by large brokers. Second, the insurance brokerage problem may be complicated by the moral hazard problem of the principal who can affect the loss distribution, and hence the actual reward made to the broker. Third, a broker may provide other tied-in riskmanagement services, such as risk analysis, claim settlement, and legal advice. This paper may be extended to cover the case of multiple agents' activities along the line of Holmstrom and Milgrom's (1991) paper in the future. Finally, reputation and long-term repeated relations between principals and agents, not taken care of in this paper, may generate optimal reward functions that would not arise in the static setting (see, e.g., Bull, 1987; MacLeod and Malcomson, 1989).

\section{Acknowledgements}

Financial support from the University Research Grants and Academic Programme Research Grants (Business, grant reference DB04A2) of Lingnan University is highly appreciated.

\section{References}

Araujo, A. and Moreira, H. (2001) 'A general Lagrangian approach for non-concave moral hazard problems', Journal of Mathematical Economics 35: 1-16.

Arrow, K.J. (1963) 'Uncertainty and the welfare economics of medical care', American Economic Review 53: 941-973.

Brown, M., Chiang, S.H., Ghost, S. and Wolfstetter, E. (1986) 'A new class of sufficient conditions for the first-order approach to principal-agent problems', Economics Letters 21: 1-6.

Bull, C. (1987) 'The existence of self-enforcing wage contracts', Quarterly Journal of Economics 102: $147-159$.

Campbell, T.S. and Kracaw, W.A. (1987) 'Optimal managerial incentive contracts and the value of corporate insurance', Journal of Financial and Quantitative Analysis 22: 315-328. 
Cummins, J.D. and Doherty, N.A. (2006) 'The economics of insurance intermediaries', Journal of Risk and Insurance 73(3): 359-396.

Grossman, S. and Hart, O. (1983) 'An analysis of the principal-agent problem', Econometrica 51: 7-45.

Holmstrom, B. (1979) 'Moral hazard and observability', Bell Journal of Economics 10: 74-91.

Holmstrom, B. and Milgrom, P. (1991) 'Multitask principal agent analyses: Incentive contracts, asset ownership and job design', Journal of Law, Economics, and Organization 7(special issue): 24-52.

Jewitt, I. (1988) 'Justifying the first-order approach to principal-agent problems', Econometrica 56: $1177-1190$.

Laffont, J. and Martimort, D. (2002) The Theory of Incentives - The Principal-agent Model, New Jersey: Princeton University Press.

LiCalzi, M. and Spaeter, S. (2003) 'Distributions for the first-order approach to principal-agent problems', Economic Theory 21: 167-173.

MacLeod, B. and Malcomson, J. (1989) 'Implicit contracts, incentive compatibility, and involuntary unemployment', Econometrica 57: 447-480.

Mirrlees, J. (1999/1975) 'The theory of moral hazard and unobservable behavior - Part I', mimeo, Nuffield College, Oxford. Published in Review of Economic Studies 66: 3-21.

Mossin, J. (1968) 'Aspects of rational insurance purchasing', Journal of Political Economy 76: $553-568$.

Oyer, P. (2000) 'A theory of sales quotas with limited liability and rent sharing', Journal of Labor Economics 18: 405-426.

Rogerson, W. (1985) 'The first-order approach to principal-agent problems', Econometrica 53: $1357-1368$.

Sinclair-Desgagné, B. (1994) 'The first-order approach to multi-signal principal-agent problems', Econometrica 62: 259-266.

Spence, M. and Zeckhauser, R. (1971) 'Insurance, information, and individual action', American Economic Review Papers and Proceedings of the Eighty-Third Annual Meeting of the American Economic Association 61: 380-387.

Tommy, V.A. (2000) 'The mystery of broker remuneration', Business Insurance 34: 25.

Whitt, W. (1980) 'Uniform conditional stochastic order', Journal of Applied Probability 17: 112-123.

\section{Appendix}

Proof of Theorem 1: To prove part (a), differentiate both sides of (5) with respect to $x$ to get

$$
v^{\prime \prime}(x-s(x))\left(1-s^{\prime}(x)\right)=\mu\left(f_{a} / f\right)_{x} .
$$

Substituting $\mu>0$ from the Lemma and $v^{\prime \prime}<0$ into (8) gives $\operatorname{sgn}\left[1-s^{\prime}(x)\right]=-\operatorname{sgn}$ $\left[\left(f_{a} / f\right)_{x}\right]$.

To prove part (b), rewrite (8) as $A_{v} \cdot\left(s^{\prime}-1\right) v^{\prime}=\mu\left(f_{a} / f\right)_{x}$ and differentiate both sides with respect to $x$ to get

$$
-A_{v^{\prime}}\left(s^{\prime}-1\right)^{2} v^{\prime}+A_{v} s^{\prime \prime} v^{\prime}-A_{v}\left(s^{\prime}-1\right)^{2} v^{\prime \prime}=\mu\left(f_{a} / f\right)_{x x} .
$$


According to (9), $s^{\prime \prime}<0$ if and only if

$$
-A_{v^{\prime}}\left(s^{\prime}-1\right)^{2} v^{\prime}-A_{v}\left(s^{\prime}-1\right)^{2} v^{\prime \prime}>\mu\left(f_{a} / f\right)_{x x} .
$$

The inequality in (10) is satisfied as $v^{\prime \prime}<0, A_{v}>0,\left(s^{\prime}-1\right)^{2} \geqslant 0, A_{v^{\prime}} \leqslant 0, \mu>0$, and $\left(f_{a} / f\right)_{x x}<0$.

Proof of Claim Suppose the MLRC is satisfied such that $\left(f_{a} / f\right)_{x} \geqslant 0, \forall x \in[x, \bar{x}]$ with $\left(f_{a} / f\right)_{x}>0$ for some subsets of $[\underline{x}, \bar{x}]$ with positive measures. Now,

$$
0=F_{a}(\bar{x}, a)=\int_{\underline{x}}^{\bar{x}} f_{a}(x, a) \mathrm{d} x
$$

implies that $f_{a} / f$ must change sign on $(\underline{x}, \bar{x})$ at least once. This together with the MLRC implies that $f_{a} / f$ must change sign exactly once. However, notice that $f_{a} / f$ may equal zero for a subset of $(\underline{x}, \bar{x})$ with positive measure. Therefore, $\exists x_{0}=\inf \left\{x \mid x \in(\underline{x}, \bar{x})\right.$ and $\left.f_{a} / f=0\right\}$ such that $f_{a} / f<0, \forall x \in\left[x, x_{0}\right)$ and $\exists x_{1}=$ $\sup \left\{x \mid x \in(\underline{x}, \bar{x})\right.$ and $\left.f_{a} / f=0\right\}$ such that $f_{a} / f>0, \forall x \in\left(x_{1}, \overline{\bar{x}}\right]$. Notice that $F_{a}(x, a)<0, \forall x \in\left(x, x_{0}\right]$. Moreover, $F_{a}(x, a)<0$ is constant on $\left[x_{0}, x_{1}\right]$. Now, consider any $x_{2} \in\left(x_{1}, \bar{x}\right)$. Rewrite (11) as

$$
\begin{aligned}
0= & F_{a}(\bar{x}, a)=\int_{\underline{x}}^{x_{0}}\left(f_{a} / f\right) f \mathrm{~d} x+\int_{x_{0}}^{x_{1}} 0 \cdot f \mathrm{~d} x \\
& +\int_{x_{1}}^{x_{2}}\left(f_{a} / f\right) f \mathrm{~d} x+\int_{x_{2}}^{\bar{x}_{a}}\left(f_{a} / f\right) f \mathrm{~d} x .
\end{aligned}
$$

Now, $f_{a} / f>0, \forall x \in\left(x_{1}, \bar{x}\right]$ implies that $\int_{x_{2}}^{\bar{x}}\left(f_{a} / f\right) f \mathrm{~d} x>0$ and hence $F_{a}\left(x_{2}, a\right)=$ $\int_{x}^{x_{2}}\left(f_{a} / f\right) f \mathrm{~d} x<0$ according to (12). Since $x_{2}$ can be chosen arbitrarily close to $x_{1}^{-}$or $\bar{x}$, one has $F_{a}(x, a)<0, \forall x \in\left(x_{1}, \bar{x}\right)$. This together with $F_{a}(x, a)<0$, $\forall x \in\left(\underline{x}, x_{1}\right]$ implies that $\int_{\underline{x}}^{x} F_{a}(\theta, a) \mathrm{d} \theta<0, \forall x \in(\underline{x}, \bar{x}]$ violating the MPSR condition at $x=\bar{x}$.

Proof of Theorem 2

As suggested by Jewitt (1988), it suffices to show that the agent's second-order condition holds, that is,

$$
\int_{\underline{x}}^{\bar{x}} s(x) f_{a a}(x, a) \mathrm{d} x-c^{\prime \prime}(a)<0 .
$$


Integrating the first term on the left side of (13) by parts twice gives

$$
\begin{aligned}
\int_{\underline{x}}^{\bar{x}} s(x) f_{a a}(x, a) \mathrm{d} x & =s(\bar{x}) \int_{\underline{x}}^{\bar{x}} f_{a a}(x, a) \mathrm{d} x-\int_{\underline{x}}^{\bar{x}} s^{\prime}(x)\left[\int_{\underline{x}}^{x} f_{a a}(\theta, a) x \theta\right] \mathrm{d} x \\
& =-\int_{\underline{x}}^{\bar{x}} s^{\prime}(x)\left[\int_{\underline{x}}^{x} f_{a a}(\theta, a) \mathrm{d} \theta\right] \mathrm{d} x \\
& =-s^{\prime}(\bar{x}) \int_{\underline{x}}^{\bar{x}} F_{a a}(x, a) \mathrm{d} x+\int^{\underline{x}} s^{\prime \prime}(x)\left[\int_{\underline{x}}^{x} F_{a a}(\theta, a) \mathrm{d} \theta\right] \mathrm{d} x \\
& \int_{\underline{x}} s^{\prime \prime}(x)\left[\int_{\underline{x}}^{x} F_{a a}(\theta, a) \mathrm{d} \theta\right] \mathrm{d} x .
\end{aligned}
$$

The second equality in (14) is because of $\int_{\underline{x}}^{\bar{x}} f_{a a}(x, a) \mathrm{d} x=F_{a a}(\bar{x}, a)=0$ (as $\left.F_{a}(\bar{x}, a)=0\right)$. The fourth equality is because of $\int_{\underline{x}}^{\bar{x}} F_{a a}(x, a) \mathrm{d} x=0$ (as $\int_{\underline{x}}^{\bar{x}} F_{a}(x, a) \mathrm{d} x=0$ for an MPSR effort). Now, conditions (b) and (c) imply that $s^{\prime \prime}<0$ according to Theorem 1 . This together with $\int_{\underline{x}}^{x} F_{a a}(\theta, a) \mathrm{d} \theta>0, \forall x \in(\underline{x}, \bar{x})$ clearly implies that the right side of the last equality of (14) is strictly negative. Therefore, (13) holds as $c^{\prime \prime}>0$. Next, replacing $f_{a a}$ and $F_{a a}$ by $f_{a}$ and $F_{a}$ in (14) and using $F_{a}(\bar{x}, a)=0$ and $\int_{\underline{x}}^{\bar{x}} F_{a}(x, a) \mathrm{d} x=0$ gives

$$
\int_{\underline{x}}^{\bar{x}} s(x) f_{a}(x, a) \mathrm{d} x=\int_{\underline{x}}^{\bar{x}} s^{\prime \prime}(x)\left[\int_{\underline{x}}^{x} F_{a}(\theta, a) \mathrm{d} \theta\right] \mathrm{d} x .
$$

$\int_{\underline{x}}^{x} F_{a}(\theta, a) \mathrm{d} \theta<0, \forall x \in(x, \bar{x})$ together with $s^{\prime \prime}<0$ implies that the right side of (15) is strictly positive. This together with $c^{\prime \prime}>0$ and $\lim _{a \rightarrow 0} c^{\prime}(a)=0$ implies that there exists $a>0$ such that the agent's first-order condition (4) holds.

\section{About the Author}

Arthur Hau has been Associate Professor of the Department of Finance and Insurance at Lingnan University. 